\title{
Motor dysfunction of the "non-affected" lower limb: a kinematic comparative study between hemiparetic stroke and total knee prosthesized patients
}

\author{
Sergio Bagnato - Cristina Boccagni - Filippo Boniforti • \\ Antonia Trinchera · Giovanni Guercio - Giulia Letizia • \\ Giuseppe Galardi
}

Received: 11 February 2008/ Accepted: 26 January 2009/Published online: 13 February 2009

(c) Springer-Verlag 2009

\begin{abstract}
In patients with hemispheric stroke, abnormal motor performances are described also in the ipsilateral limbs. They may be due to a cortical reorganization in the unaffected hemisphere; moreover, also peripheral mechanisms may play a role. To explore this hypothesis, we studied motor performances in 15 patients with hemispheric stroke and in 14 patients with total knee arthroplasty, which have a reduced motility in the prosthesized leg. Using the unaffected leg, they performed five superimposed circular trajectories in a prefixed pathway on a computerized footboard, while looking at a marker on the computer screen. The average trace error was significantly different between the groups of patients and healthy subjects $\left[F_{(2,25)}=7.9\right.$; $p=0.003]$; on the contrary, the test time execution did not vary significantly. In conclusion, both groups of patients showed abnormal motor performances of the unaffected leg; this result suggests a likely contribution of peripheral mechanisms.
\end{abstract}

Keywords Stroke $\cdot$ Knee arthroplasty . Ipsilateral motor impairment $\cdot$ Hemiparesis

\footnotetext{
S. Bagnato · C. Boccagni - A. Trinchera - G. Guercio ·

G. Galardi $(\bowtie)$

Rehabilitation Department,

Fondazione Istituto San Raffaele-G.Giglio,

90015 Cefalù (PA), Italy

e-mail: giuseppe.galardi@hsrgiglio.it

F. Boniforti

Orthopaedic Department,

Fondazione Istituto San Raffaele-G. Giglio,

Cefalù (PA), Italy

G. Letizia

Physical Medicine and Rehabilitation Department,

University of Palermo, Palermo, Italy
}

\section{Introduction}

Several reports demonstrated an abnormal function of ipsilateral limbs in hemispheric stroke patients [1-3]. It has been documented in both upper [1, 2, 4, 5] and lower limbs [6] and it was related to strength [7], speed and dexterity [8], temporal coordination and complex manual task execution [9].

The reasons of this dysfunction are still debated and may be due to multiple factors. It may be caused by cognitive deficits affecting perception and control of action. In patients with left hemisphere damage, ipsilateral motor dysfunction may be due to ideomotor apraxia, while in patients with right hemisphere injury, it may be caused by visuospatial impairments $[8,10]$. However, impaired ipsilateral function has also been reported in patients without cognitive impairment $[2,3]$.

The abnormal ipsilateral descending output, which is associated with an unilateral cortical lesion, may contribute to ipsilateral limbs abnormal function too [10-12]. The presence of a bilateral component in motor control is supported by the anatomical evidences of a small ipsilateral component into the corticospinal tract [13] and by observations that a functional activation might have a bilateral expression in cortical areas [14]. Also, spinal reflexes abnormalities might contribute to spread abnormal motor command to the ipsilateral side. Actually, spinal reflexes can show bilateral long-lasting changes in hemiparetic subjects [15]. In stroke patients descending commands, by interacting with a network of spinal interneurons and motoneurons, might cause an abnormal level of excitability in both sides. Thus, the combination of abnormal descending commands and altered spinal excitability might be responsible for the ipsilateral motor impairment. 
Moreover, in patients with stroke other impairments of the affected side, such as weakness, loss of weight sense, pain, immobilization, etc., might be accounted for "other side leg" motor impairment. About this topic, there is a reduced number of studies underlining the role of peripheral mechanisms [2]. For instance, an abnormal postural stabilization too may play a role in ipsilateral motor impairment $[3,16]$.

To explore the role of peripheral mechanisms in the genesis of ipsilateral motor limb abnormalities, we matched two different groups of patients with unilateral leg impairment, respectively, caused by a cerebral lesion and by a joint lesion. Thus the two groups of patients were chosen because they had an impaired motility of the affected leg as a result of stroke and knee prosthesis. In both groups we may hypothesize, as common peripheral factor, the presence of a reduced flow of sensory afferents due to an impaired motility of the affected leg. We measured the motor function of the "other side leg" in stroke patients and in patients with recent total knee arthroplasty by using a computerized proprioceptive kinematic footboard (Pro-Kin system, Tecnobody, Italy). Results were compared with those obtained in healthy subjects.

\section{Methods}

\section{Participants}

Three groups of subjects have been included: patients with hemiparesis caused by stroke (HS), patients with total knee arthroplasty (TKA) and control subjects (CS). None of the participants used neuroactive drugs; patients affected by diabetes, polineuropathy and mielopathy were excluded.

\section{HS group}

Out of the 15 patients initially included in the study, 5 were excluded later due to mistakes in the execution of the test or to its early interruption. Out of the 10 patients, who performed a correct test, 7 were men and 3 women (mean age $64.4 \pm 6.4$ years) (see Table 1). All patients were at their first stroke event and they presented a severe spastic hemiparesis, with absence or just minimal motor activity in the arm and a residual strength not more than 3 at the Medical Research Council (MRC) scale in the leg. A thorough neurological examination was performed in all patients, without evidence of deficit of strength, sensation and apraxia in the ipsilateral limbs. Moreover, no patient had spatial impairments. All of them had an adequate trunk control, so that they were able to maintain a comfortable seated position during the text execution and were able to walk for some meters with assistive devices. No synkinetic movements were present during the test execution. Patients participated to the study as long as no relevant cognitive (mini mental state examination $>25 / 30$ ) and visual impairments were present. Neuroimaging studies did not show any lesion in the non-affected hemisphere. The interval time from the acute event to the experiments was $6.8 \pm 6.2$ months.

\section{TKA group}

Out of the 14 patients initially included in the study, 6 were excluded later due to mistakes in the execution of the test or to its early interruption. Out of the 8 patients, who performed a correct test, 4 were men and 4 women (mean age $65 \pm 3.9$ years). Four had a right TKA and 4 had a left TKA. All patients were right handed. None had experienced other joint or bone diseases and all of them were able to walk before surgery. At the time of the examination, no sensory disturbances or pain in the affected limb were referred; a standard nerve conduction and electromyographic study was performed in all patients to exclude post surgery nervous lesions. The functional normality of the unaffected side was evaluated by an orthopaedic history and clinical examination and by X-rays, without evidence
Table 1 Demographic and clinical characteristics of the 10 patients with stroke who performed the test correctly

$F$ female, $M$ male, $R$ right, $L$ left, $M C A$ middle cerebral artery

\begin{tabular}{llllll}
\hline Patient & Gender & Age & Handed & $\begin{array}{l}\text { Time after } \\
\text { stroke (months) }\end{array}$ & Lesion \\
\hline 1 & $\mathrm{~F}$ & 68 & $\mathrm{R}$ & 5 & L MCA infarction (cortical) \\
2 & $\mathrm{~F}$ & 59 & $\mathrm{R}$ & 2 & L MCA infarction (cortical) \\
3 & $\mathrm{M}$ & 63 & $\mathrm{R}$ & 6 & L Thalamocapsular infarction \\
4 & $\mathrm{M}$ & 61 & $\mathrm{R}$ & 8 & L MCA infarction (cortical-subcortical) \\
5 & $\mathrm{M}$ & 62 & $\mathrm{R}$ & 2 & R Thalamocapsular infarction \\
6 & $\mathrm{~F}$ & 65 & $\mathrm{R}$ & 22 & R MCA infarction (cortical) \\
7 & $\mathrm{M}$ & 60 & $\mathrm{R}$ & 12 & R MCA infarction (cortical) \\
8 & $\mathrm{M}$ & 76 & $\mathrm{R}$ & 3 & L MCA infarction (cortical-subcortical) \\
9 & $\mathrm{M}$ & 56 & $\mathrm{R}$ & 6 & R MCA infarction (cortical) \\
10 & $\mathrm{M}$ & 74 & $\mathrm{R}$ & 2 & L MCA infarction (cortical-subcortical) \\
\hline
\end{tabular}


of sensory-motor abnormalities. The hip, knee and ankle arthritic changes were graded less than II [17]. All patients were passively mobilized since the day after surgery; they were able to flex the knee of $110^{\circ}$ in order to put their foot on the plank behind the footboard and to stabilize the trunk during the test execution. Time from surgery to the evaluation was $6.1 \pm 3.7$ days.

\section{CS group}

Ten subjects, 6 men and 4 women (mean age $61.4 \pm 7.5$ years). They were patients close relatives. All subjects were right handed and in good health. Tests were performed using the right or the left foot in a randomized way (in 6 and in 4 subjects, respectively); none of the CS was excluded because of mistakes or early interruption.

CS and patients gave their informed consent according to the Declaration of Helsinki.

\section{ProKin system}

The system is an electronic transducer board swinging on a pivot that allows angular movements in sagittal and frontal planes. When the foot of the patients moves the footboard, data are transmitted from the transducers to an analogic/ digital converter and sent to the PC. The software processes data and shows in real time traces of the mobile footboard movement on the computer screen.

All subjects were seated on a comfortable chair, adjustable in height, to allow horizontal position of the thigh, knee bent at $110^{\circ}$, ankle plantar flexed at $10^{\circ}$. The foot was kept on the centre of the footboard and the affected leg on the plank apart the footboard in a rest position (Fig. 1). The head was kept in primary position, to allow the subjects to comfortably watch the monitor.

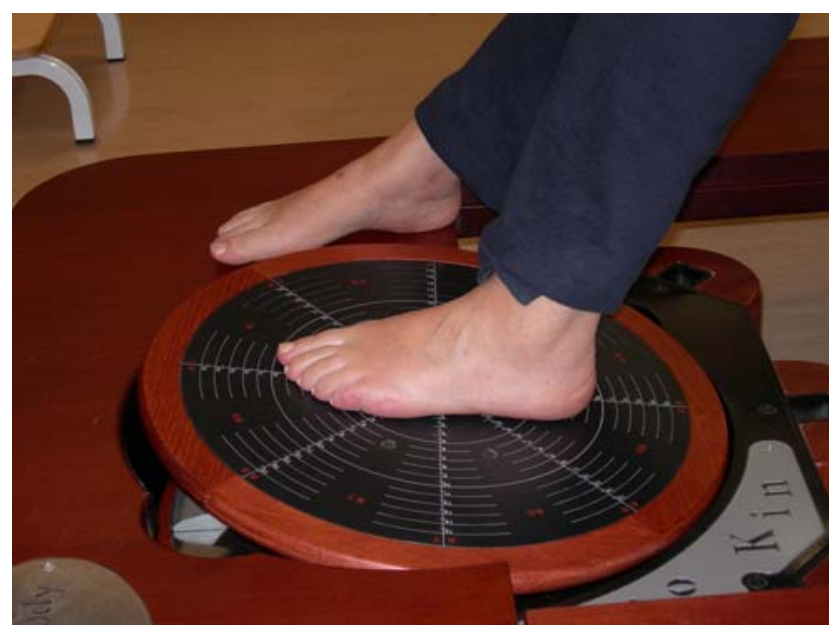

Fig. 1 Position of the foot on the footboard
The experimental protocol consisted of two consecutive phases: the training and the evaluation.

The training phase was applied to gain confidence with the system; it consisted of three series of exercises. The subject was asked to use his/her lower limb to follow consecutive vertical (Fig. 2a), horizontal (Fig. 2b) and oblique (Fig. 2c) sequences, each one lasting 120 s. They moved the cursor on the screen tilting the board and tracing the route with an active movement of the foot and inverting direction when the target was signalled. The exercise stopped automatically at the end of the $120 \mathrm{~s}$. There was an interval time of $5 \mathrm{~min}$ between each series.

The evaluation phase. The subject was asked to use his/her lower limb to follow a circular route in a predefined corridor (Fig. 2d) five consecutive times. A bell rang anytime the cursor crossed the border. The direction of the exercise was clockwise if the right limb was used and anti-clockwise if the left one was used. To follow the requested route the board had to be tilted at $7.5^{\circ}$ in each plane. The test stopped automatically at the end of the five turns. Patients had to perform the tests as quickly as possible and to the best of their ability. Mistakes or interruptions before the end caused the exclusion of the subject from the study to avoid the possibility of a training effect if the exercises were repeated. The experimental session (training and evaluation phases) lasted for about 25 min and was well tolerated by all patients.

The average trace error (ATE) and time to carry out the test time execution (TTE) were evaluated.

The ATE was calculated for each of the five turns required in absolute value, without positive or negative signs, as ATE $=\frac{(\mathrm{OP}-\mathrm{RP})}{\mathrm{RP}} * 100$, where OP was the length of the observed path (executed by subjects), RP was the length of the requested (ideal) path. Large ATE values mean large errors in path control, 0 means a perfect task execution (the trace of the patient's path could perfectly overlap the requested path). TTE was the time (in seconds) from the start to the end of the trial.

Statistical analysis

For the studied variables all data are expressed as mean \pm standard deviation (SD). A one-way analysis of variance (ANOVA) was used to compare each variable between CS, HS and TKA patients. When appropriate, Tukey's honest significant difference test was used for post hoc analysis.

\section{Results}

The ATE was $55.9 \pm 18.7$ for CS, $95.6 \pm 34.1$ for HS and $104.2 \pm 33.0$ for TKA (Fig. 3). ANOVA showed that the ATE was significantly different between CS, HS and TKA $\left(F_{(2,25)}=7.9 ; p=0.003\right)$. In particular, post hoc $t$ tests 
Fig. 2 The trajectories that patients and control subjects had to follow on the screen moving their foot. $\mathbf{a}, \mathbf{b}$ and $\mathbf{c}$ describe the exercises of the training phase. $\mathbf{d}$ is the evaluation test in which subjects had to trace on the screen 5 circular trajectories superimposed on the midline (the ideal line); the arrow is the cursor that subjects had to move
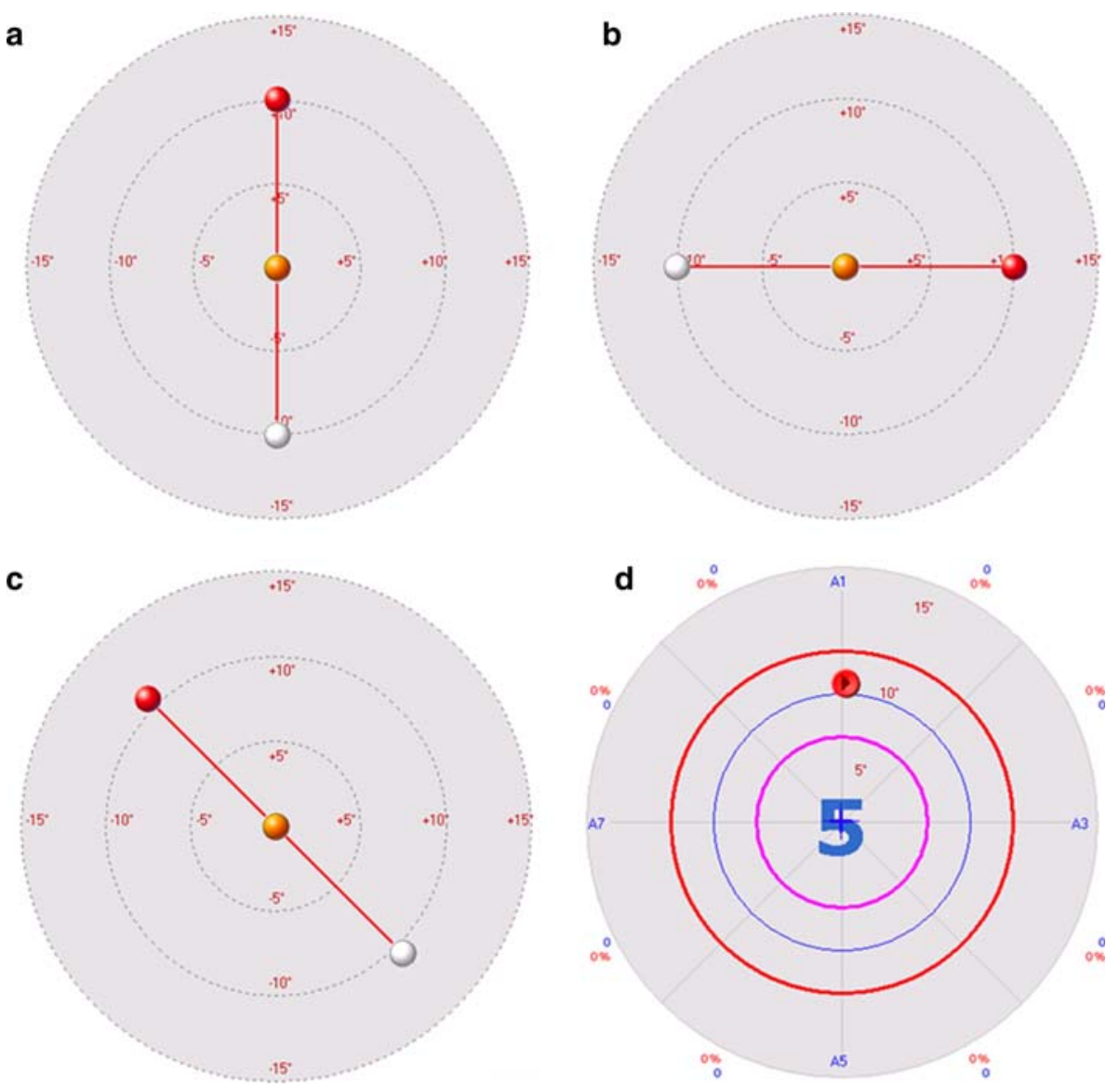

revealed that ATE was significantly greater in HS $(p=0.01)$ and TKA patients $(p=0.005)$ compared to CS (Fig. 3). Figure 4 reports examples of the traces in one CS and in one HS and TKA patient.

The TTE was $39.7 \pm 15.9 \mathrm{~s}$ in CS, $65.9 \pm 29.3 \mathrm{~s}$ in HS and $51.6 \pm 25.6 \mathrm{~s}$ in TKA (Fig. 5). No significant difference was found between the three groups $\left(F_{(2,25)}=2.9\right.$; $p=0.07)$.

Six HS patients had parameters exceeding the cut-off of two standard deviations from mean normal value: three exceeded in both ATE and TTE, two in ATE alone and one in TTE alone. Of these patients, two had right hemiparesis and four left hemiparesis.

Six TKA patients had parameters exceeding the cut-off of two standard deviations from mean normal value: one exceeded in both ATE and TTE, four in ATE alone and one in TTE alone. Of these patients, three have had right knee replacement surgery and three left.

\section{Discussion}

This study has been designed in order to understand the mechanisms responsible of ipsilateral leg motor impairment in patients with stroke and, particularly, to evaluate

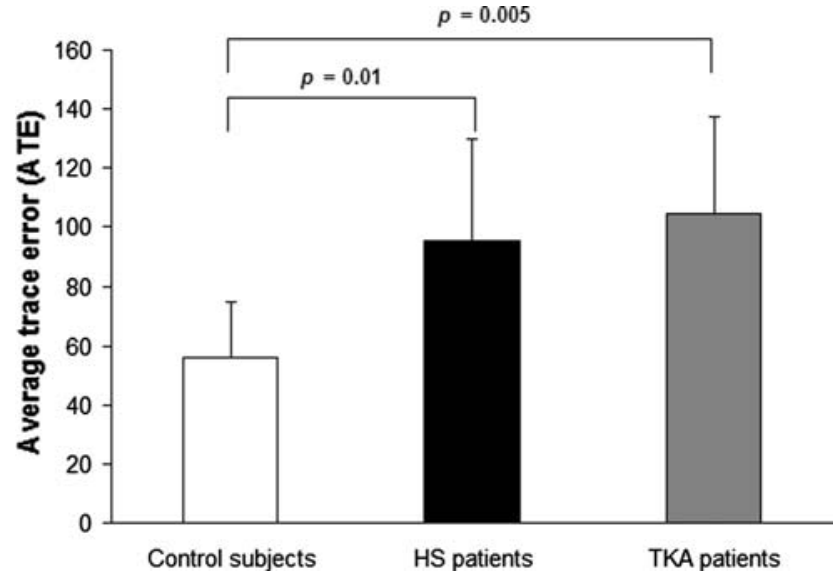

Fig. 3 Average trace error (ATE): mean value and SD. The ATE was calculated as ATE $=\frac{(\mathrm{OP}-\mathrm{RP})}{\mathrm{RP}} * 100$, where OP was the length of the observed path (executed by patients), RP was the length of the requested (ideal) path

the possible contribute of peripheral mechanisms. We adopted the term "other side leg" to indicate the "normal limbs" of both groups of patients in order to avoid misunderstanding between "ipsilateral limb" used in HS patients and "contralateral limb" in TKA. TKA group was chosen since the motor disability of the "other side leg" 


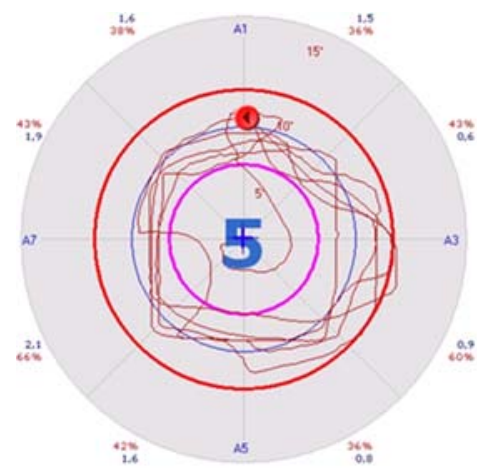

a

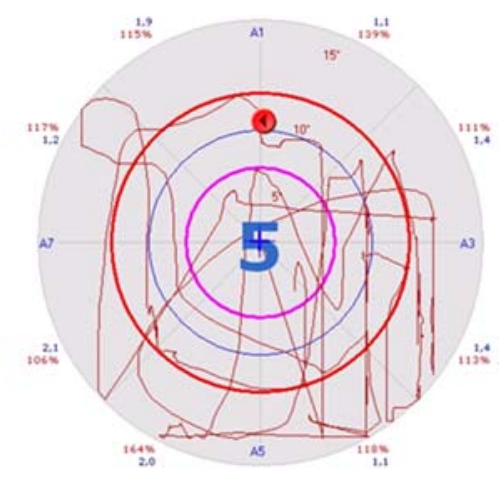

b

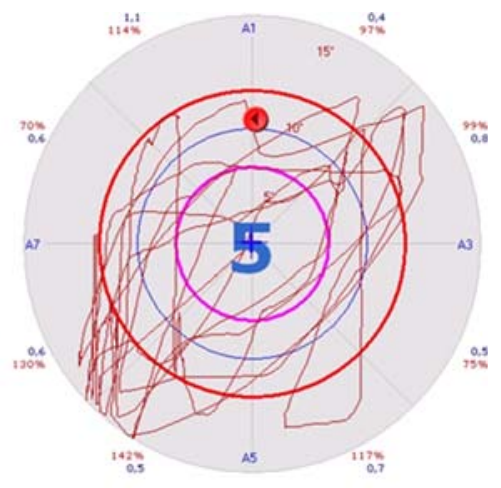

C

Fig. 4 Evaluation test. Example the traces in one control subject (a), in one hemiparetic patient (b) and in one patient with knee replacement surgery (c)

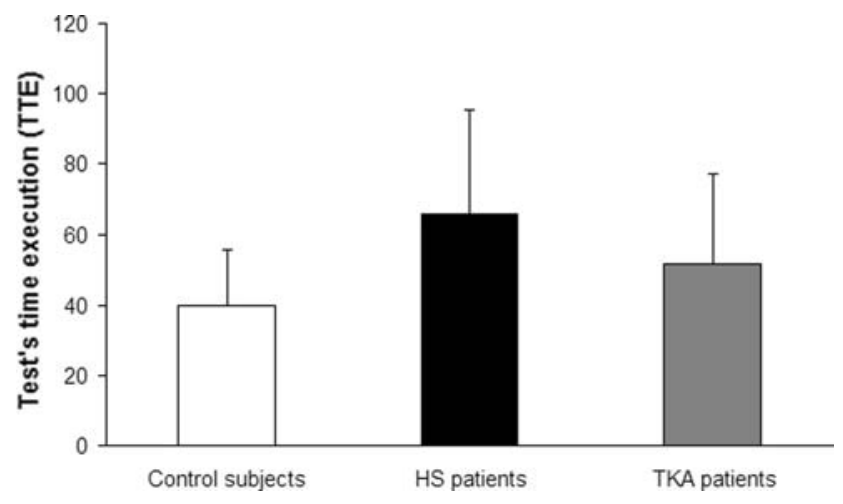

Fig. 5 Test time execution (TTE) from the start to the end of the trials: mean values (in seconds) and SD

might be caused by an abnormal sensory afferents flow due to reduced motility in the prosthesized leg. In HS group, the expected motor disability of the "other side leg" might instead be caused both by the lesion in the contralateral hemisphere and by the abnormal sensory afferents due to the reduced motility resulting from the paresis.

Both HS and TKA patients exhibited abnormal motor performances of the "other side leg".

In this study, we found the following: (a) a significant increment of the ATE, (b) an increment of the TTE, although without statistical significance, (c) a number of patients in which the ATE and the TTE exceeded the cutoff of two standard deviation from normal values and (d) an high percentage of patients who were unable to conclude the test. Since clinical evaluation did not show neuromuscular deficits, functional errors, such as mistakes in direction and amplitude, differences in velocity and acceleration, failure to control the inertial properties of the limb in the initial trajectory may be accounted for these results. These results suggest that the impaired sensory afferents flow play a determinant role in TKA and a likely concurrent role in HS in the abnormal test execution of the "other side leg". Abnormalities in the peripheral afferent input or in the brain response to sensory input may interfere with motor programs processing in the cortical motor areas. We cannot state if this result correlates, in HS patients, with specific sites of the cerebral lesion or with the phase of disease (acute, sub-acute or chronic stroke) and if, in all patients, it is due to a specific cinematic alteration (peak of velocity, acceleration, etc.). It would be interesting to be able to evidence a typical pattern of movement abnormality in HS and TKA patients. However, the test used in our study could highlight only an overall error rate; so, further studies are necessary in order to answer to this question.

Due to the nature of the TKA patients disability, mechanisms such as cognitive deficits [8, 10], abnormal ipsilateral descending output and spinal reflexes [10-12, $15,18]$ have not been discussed. On the contrary, reduced motility and proprioceptive impairments are themes of discussion which involve both groups of patients. Motor impairment of the "other side leg" may be a consequence of abnormal postural stabilization [3, 16]. In HS patients, the force produced by the "ipsilateral limb" is reduced because of the difficulty in supplying contralateral stabilization using the paretic side owing to weakness, spasticity and abnormal postures [19]. This difficulty might explain the deficits observed in functional tasks such as gross and fine dexterity, global performance and motor coordination [20]. However, this abnormal postural stabilization is unlikely in TKA patients, in whom the trunk muscle control operates in order to compensate the actual loading deficit of the immobilized leg. Actually, TKA patients were able to put their affect ed foot on the plank behind the footboard, in order to stabilize the "other side leg" during the text execution. It is possible to suppose that the abnormal performances of the "other side leg" in TKA patients are a consequence of the reduced motility, with a loss of sensory afferents from the affected leg. This reduced flow of sensory afferents, due to an impaired 
motility, is a "peripheral" factor present also in HS patients. More likely, abnormal motor performances in both group of patients were due to the involvement of complex mechanisms, requiring the integrity of both hemispheres and suitable information from the periphery. Gordon et al. [21] reported impairments of reaching movements in patients without proprioception. Ting et al. [22] outlined how the sensory state of the contralateral leg may affect ipsilateral muscle coordination of pedaling movements. In both groups of patients, afferent inputs from muscles, tendons and capsular joints were altered due to their reduced motility. Moreover, patients with stroke may have motor and sensitive impairments in "contralateral limb" which may interfere with motor control of the ipsilateral one; likewise, patients with TKA may have abnormal proprioception as a consequence of the surgical introduction of a prosthetic joint [23, 24], which may interfere with motor control of the contralateral one. The disruption of the sensory afferents to central nervous system may be the trough for the "other side" abnormal motor performances by inducing changes into cerebral connectivity [25]. It is well known that sensory information from the peripheral receptors, the continuous feedback process at the spinal level and their central processing, may play an important regulatory role on motor performances [26-28].

In this way, the abnormal performances of the "other side leg", plausibly acting on central nervous system level, might be seen as the core of a neuroplastic process, produced by both central and peripheral causes. In HS patients, the involvement of the intact hemisphere may be due to the contralateral hemispheric lesion as well as to the sensory loss of peripheral afferents, caused by the reduced motility; in TKA patients, the involvement of the ipsilateral hemisphere, by means of the contralateral one, may be due only to a loss of sensory afferents [29]. Therefore, in both groups of patients, it is possible to hypothesize that a reorganization in the ipsilateral hemisphere may be crucial to explain the altered performances in our test. A surprising finding is that TKA patients, in whom only peripheral mechanisms should operate, have similar or slightly increased values of ATE compared with HS patients. We may formulate some hypothesis to explain this result: (a) HS patients may have various neuroplastic compensatory strategies during the test execution, because they are studied months after the stroke, unlike TKA patients, who are studied some days after the surgery; (b) there is a predominant role for the peripheral mechanisms also in HS patients during the test execution.

Animal studies demonstrated that a neocortical infarction leads to hyper-excitability in contralateral hemisphere, contributing to a functional reorganization after stroke [30, 31]. In humans, neurophysiological studies showed an intact hemisphere increased excitability [32], likely due to a transhemispheric diaschisis [33]. Moreover, functional MRI studies confirmed an increased activation of ipsilateral motor areas in patients after stroke, while moving the affected limb [34-36]. Another possible mechanism is that damage in one hemisphere may alter trans-callosal signals and disrupt neural processing in the opposite one. In humans, the transient deafferentation by means of regional anaesthesia or ischemic nerve block induces cortical changes such as modifications in the amplitude of motor evoked potentials and in regional cerebral blood flow [37, 38]. This cortical reorganization may imply an adaptive change to compensate for motor deficits or it may be the expression of the unmasking of normally inhibited pathways [39], even if its meaning in stroke patients is still unclear. These experimental observations lead us to suggest that a loss of the normal pattern of interactions between the affected hemisphere and the non-affected one could underlie the abnormal motor performance in the "other side leg".

In conclusion, this study might introduce new insights into: (1) the role of peripheral sensory input in the interhemispheric connectivity, (2) the pathophysiology of the "other side leg" altered performances and (3) the rehabilitative programs to achieve the best recovery. In fact, it is well known that early rehabilitation prevents complications such as skin sores, joint rigidity and muscular stiffness; moreover, it might contribute actively to the recovery and to avoid the "other side leg" abnormalities by conveying sensory information to cortical motor areas, by facilitating the sensory-motor integration and the processes of neuronal plasticity and by sustaining the execution of motor programs. Furthermore, our results suggest to extend the attention to the "other side" in stroke patients as well as in other kind of unilateral motor impairment in order to achieve better rehabilitative results [40].

Acknowledgment The authors thank Davide Cattaneo, Pht, from Fondazione Don Gnocchi of Milan, for his contribution on technical and methodological aspects of this study.

\section{References}

1. Jebsen RH, Griffith ER, Long EW, Fowler R (1971) Function of the "normal" hand in stroke patients. Arch Phys Med Rehabil 52:170-174

2. Jones RD, Donaldson IM, Parkin PJ (1989) Impairment and recovery of ipsilateral sensory-motor function following unilateral cerebral infarction. Brain 112:113-132

3. Desrosiers J, Bourbonnais D, Bravo G, Roy PM, Guay M (1996) Performances of the "unaffected" upper extremity of elderly stroke patients. Stroke 27:1564-1570

4. Colebatch JG, Gandevia SC (1989) The distribution of muscular weakness in upper motor neuron lesions affecting the arm. Brain 112:749-763

5. Smutok MA, Grafman G, Salazar AM, Sweeney JK, Jonas BS, DiRocco PJ (1989) Effects of unilateral brain damage on 
contralateral and ipsilateral upper extremity function in hemiplegia. Phys Ther 69:195-203

6. Kim SH, Pohl PS, Luchies CW, Stylianou AP, Won Y (2003) Ipsilateral deficits of targeted movements after stroke. Arch Phys Med Rehabil 84:719-724

7. Andrews AW, Bohannon RW (2000) Distribution of muscle strength impairments following stroke. Clin Rehabil 14:79-87

8. Sunderland A, Bowers MP, Sluman S-M, Wilcock DJ, Ardron ME (1999) Impaired dexterity of the ipsilateral hand after stroke and the relationship to cognitive deficit. Stroke 30:949-955

9. Yelnik A, Bonan I, Debray M, Lo E, Gelbert F, Bussel B (1996) Changes in the execution of a complex manual task after ipsilateral ischemic cerebral hemispheric stroke. Arch Phys Med Rehabil 77:806-810

10. Winstein C, Pohl P (1995) Effects of unilateral brain damage on the control of goal-directed hand movements. Exp Brain Res 105:163-174

11. Carey JR, Baxter TL, Di Fabio RP (1998) Tracking control in the nonparetic hand of subjects with stroke. Arch Phys Med Rehabil 79:435-441

12. Hermsdorfer J, Laimgruber K, Kerkhoff G, Mai N, Goldenberg G (1999) Effects of unilateral brain damage on grip selection, coordination, and kinematics of ipsilesional prehension. Exp Brain Res 128:41-51

13. Nathan PW, Smith MC, Deacon P (1990) The corticospinal tracts in man. Course and location of fibres at different segmental levels. Brain 113:303-324

14. Cramer SC (1999) Stroke recovery. Lessons from functional MR imaging and other methods of human brain mapping. Phys Med Rehabil Clin N Am 10:875-886

15. Dewald JP, Beer RF, Given JD, McGuire JR, Rymer WZ (1999) Reorganization of flexion reflexes in the upper extremity of hemiparetic subjects. Muscle Nerve 22:1209-1221

16. Bertrand AM, Mercier C, Shun PL, Bourbonnais D, Desrosiers J (2004) Effects of weakness on symmetrical bilateral grip force exertion in subjects with hemiparesis. J Neurophysiol 91:15791585

17. Petersson IF, Boegard T, Saxne T, Silman AJ, Svensson B (1997) Radiographic osteoarthritis of the knee classified by the Ahlback and Kellgren \& Lawrence systems for the tibiofemoral joint in people aged 35-54 years with chronic knee pain. Ann Rheum Dis 56:493-496

18. Thilmann AF, Fellows SJ, Garms E (1991) The mechanism of spastic muscle hypertonus. Variation in reflex gain over the time course of spasticity. Brain 114:233-244

19. Gauthier J, Bourbonnais D, Filiatrault J, Gravel D, Arsenault AB (1992) Characterization of contralateral torques during static hip efforts in healthy subjects and subjects with hemiparesis. Brain 115:1193-1207

20. Massion J (1992) Movement, posture and equilibrium: interaction and coordination. Prog Neurobiol 38:35-56

21. Gordon J, Ghilardi MF, Ghez C (1995) Impairments of reaching movements in patients without proprioception I. Spatial errors. J Neurophysiol 73:347-360

22. Ting LH, Raasch CC, Brown DA, Kautz SA, Zajac FE (1998) Sensorimotor state of the contralateral leg affects ipsilateral muscle coordination of pedaling. J Neurophysiol 80:1341-1351
23. Pap G, Meyer M, Weiler HT, Machner A, Awiszus F (2000) Proprioception after total knee arthroplasty: a comparison with clinical outcome. Acta Orthop Scand 71:153-159

24. Wada M, Kawahara H, Shimada S, Miyazaki T, Baba H (2002) Joint proprioception before and after total knee arthroplasty. Clin Orthop Relat Res 403:161-167

25. Hanlon CA, Buffington AL, McKeown MJ (2005) New brain networks are active after right MCA stroke when moving the ipsilesional arm. Neurology 64:114-120

26. Hasan Z (1992) Role of proprioceptors in neural control. Curr Opin Neurobiol 2:824-829

27. Pearson KG (1995) Proprioceptive regulation of locomotion. Curr Opin Neurobiol 5:786-791

28. Devanne H, Maton B (1998) Role of proprioceptive information in the temporal coordination between joints. Exp Brain Res 119:58-64

29. Zanette G, Manganotti P, Fiaschi A, Tamburin S (2004) Modulation of motor cortex excitability after upper limb immobilization. Clin Neurophysiol 115:1264-1275

30. Buchkremer-Ratzmann I, August M, Hagemann G, Witte OW (1996) Electrophysiological transcortical diaschisis after cortical photothrombosis in rat brain. Stroke 27:1105-1111

31. Buchkremer-Ratzmann I, Witte OW (1997) Extended brain disinhibition following small photothrombotic lesions in rat frontal cortex. NeuroReport 8:519-522

32. Butefisch CM, Netz J, Wessling M, Seitz RJ, Homberg V (2003) Remote changes in cortical excitability after stroke. Brain 126:470-481

33. Andrews RJ (1991) Transhemispheric diaschisis. A review and comment. Stroke 22:943-949

34. Cramer SC, Nelles G, Benson RR et al (1997) A functional MRI study of subjects recovered from hemiparetic stroke. Stroke 28:2518-2527

35. Cao Y, D'Olhaberriague L, Vikingstad EM, Levine SR, Welch KM (1998) Pilot study of functional MRI to assess cerebral activation of motor function after post-stroke hemiparesis. Stroke 29:112-122

36. Jaillard A, Martin CD, Garambois K, Lebas JF, Hommel M (2005) Vicarious function within the human primary motor cortex? A longitudinal fMRI stroke study. Brain 128:1122-1138

37. Brasil-Neto JP, Cohen LG, Pascual-Leone A, Jabir FK, Wall RT, Hallett M (1992) Rapid reversible modulation of human motor outputs after transient deafferentation of the forearm: a study with transcranial magnetic stimulation. Neurology 42:1302-1306

38. Sadato N, Zeffiro TA, Campbell G, Konishi J, Shibasaki H, Hallett M (1995) Regional cerebral blood flow changes in motor cortical areas after transient anesthesia of the forearm. Ann Neurol 37:74-81

39. Manganotti P, Patuzzo S, Cortese F, Palermo A, Smania N, Fiaschi A (2002) Motor disinhibition in affected and unaffected hemisphere in the early period of recovery after stroke. Clin Neurophysiol 113:936-943

40. Pohl PS, Winstein CJ (1999) Practice effects on the less-affected upper extremity after stroke. Arch Phys Med Rehabil 80:668-675 